\title{
A Rate-Based Borrowing Scheme for QoS Provisioning in Multimedia Wireless Networks
}

\author{
Mona El-Kadi, Stephan Olariu, Member, IEEE, and Hussein Abdel-Wahab, Senior Member, IEEE
}

\begin{abstract}
Now that cellular networks are being called upon to support real-time interactive multimedia traffic such as video teleconferencing, these networks must be able to provide their users with quality-of-service (QoS) guarantees. Although the QoS provisioning problem arises in wireline networks as well, mobility of hosts, scarcity of bandwidth, and channel fading make QoS provisioning a challenging task in wireless networks. It has been noticed that multimedia applications can tolerate and gracefully adapt to transient fluctuations in the QoS that they receive from the network. The management of such adaptive multimedia applications is becoming a new research area in wireless networks. As it turns out, the additional flexibility afforded by the ability of multimedia applications to tolerate and adapt to transient changes in the QoS parameters can be exploited by protocol designers to significantly improve the overall performance of wireless systems. The main contribution of this paper is to propose a novel, rate-based, borrowing scheme for QoS provisioning in high-speed cellular networks carrying multimedia traffic. Our scheme attempts to allocate the desired bandwidth to every multimedia connection originating in a cell or being handed off to the cell. The novelty of our scheme is that, in case of insufficient bandwidth, in order not to deny service to requesting connections (new or hand-off), bandwidth will be borrowed, on a temporary basis, from existing connections. Our borrowing scheme guarantees that no connection gives up more than its fair share of bandwidth, in the sense that the amount of bandwidth borrowed from a connection is proportional to its tolerance to bandwidth loss. Importantly, our scheme ensures that the borrowed bandwidth is promptly returned to the degraded connections. Extensive simulation results show that our rate-based QoS provisioning scheme outperforms the best previously known schemes in terms of call dropping probability, call blocking probability, and bandwidth utilization.
\end{abstract}

Index Terms-Bandwidth allocation, cellular networks, QoS provisioning, multimedia traffic, reservation schemes, hand-off management, admission control, rate-based fairness.

\section{INTRODUCTION}

W E are witnessing an unprecedented demand for wireless networks to support both data and real-time multimedia traffic. While best-effort service suffices for datagram traffic, the usability of real-time multimedia applications is vastly improved if the underlying network can provide adequate quality-of-service (QoS) guarantees. Admission control and bandwidth allocation schemes can offer wireline networks the ability to provide their users with such guarantees. Due to host mobility, scarcity of bandwidth, and an assortment of channel impairments, the QoS provisioning problem is far more challenging in wireless networks than in their wireline counterparts. For example, a mobile host may be admitted into the network in a cell where its needs can easily be met, but the mobile host may eventually move to a cell that has little or no resources to offer. Since the user's itinerary and the availability of resources in various cells is usually not known in advance, global QoS guarantees are very hard to provide [1], [13].

Admission control refers to the task of deciding if a connection should be admitted into and supported by the network. Admission control is necessary for real-time, continuous media connections since the amount of resources requested by these connections may not match the level of resources available at the time of connection

- The authors are with the Department of Computer Science, Old Dominion University, Norfolk, VA 23529-0162. E-mail: olariu@cs.odu.edu.

Manuscript received 27 Mar. 2001; accepted 18 June 2001.

For information on obtaining reprints of this article, please send e-mail to: tpds@computer.org, and reference IEEECS Log Number 113888. setup [16]. Admitting a connection into the network is tantamount to a contract between the network and the connection: On the one hand, the network guarantees that a certain level of resources will be maintained for the duration of the connection. On the other hand, the connection is expected not to request additional resources over and above those negotiated at connection setup. The agreed-upon amount of resources that the network guarantees to a connection is commonly referred to as QoS. Traditional QoS parameters include bandwidth, end-to-end delay, and jitter. However, there are some QOS parameters that are specific to wireless networks.

It is typical in most admission schemes to deny service to a new connection whose requests for resources cannot be met by the network. In such a case, the connection ${ }^{1}$ is said to be blocked. In cellular networks, an important QoS parameter is the call blocking probability (CBP), denoting the likelihood that a new connection request will be denied admission into the network. A similar situation arises when an established connection in one cell attempts to migrate into a neighboring cell (i.e., a hand-off is attempted). If the new cell cannot support the level of resources required by the connection, the hand-off is denied and the connection is dropped. The call dropping probability (CDP) expresses the likelihood that an existing connection will be forcibly terminated during a hand-off between cells due to a lack of resources in the target cell. The CBP and CDP together offer a good indication of a network's quality of service in

1. We will follow common practice and refer to connections as "calls." 
the face of mobility. An additional important consideration is the degree to which the network makes an effective use of bandwidth-unquestionably its most scarce resource. This parameter, referred to as bandwidth utilization, expresses the ratio between the amount of bandwidth used by various applications admitted into the network and either the total bandwidth requested or the total bandwidth available, whichever is smaller. Keeping the CBP and CDP low while at the same time maximizing bandwidth utilization is one of the most challenging tasks facing protocol designers [11], [12], [13], [14], [20].

The traditional admission control process outlined above is, in many cases, too conservative and pessimistic. Indeed, multimedia applications are known to be able to tolerate and adapt to transient fluctuations in QoS [5], [9], [15]. This adaptation is typically achieved by the use of an adjustablerate codec or by employing hierarchical encoding of voice and/or video streams [5], [9], [18], [19]. The codec, along with appropriate buffering before play-out, can allow applications to gracefully adapt to temporary bandwidth fluctuations with little or no perceived degradation in overall quality. The graceful adaptation of applications to transient fluctuations in QoS is fundamental in wireless networks, where QoS provisioning is a very challenging task. As we shall demonstrate in this paper, the additional flexibility afforded by this ability to adapt can be exploited by protocol designers to significantly improve the overall performance of wireless systems.

As we briefly mentioned, once a connection is admitted into the network, resources must be allocated, at the negotiated level, for the duration of the connection. It is important to realize that, in a cellular network where the user may move through the network traversing a sequence of cells, this commitment cannot be only local to the cell in which the connection originated. If the connection is to be maintained after the user crosses the boundary between neighboring cells (i.e., after a hand-off), the network must guarantee an appropriate level of resources in each new cell that the user traverses. Without detailed knowledge about the intended destination of each connection, honoring this commitment is a very difficult task indeed [7], [10], [17].

\subsection{Hand-Off Prioritization Schemes}

It is well known that, from the end-user's perspective, an initial blocking of a connection attempt is more acceptable than to force-terminate an ongoing connection due to lack of resources during hand-off [2], [3], [4], [6], [8]. There are several ways in which one can ensure that hand-off connections are given priority over new call attempts in congested cells. Some of these strategies involve:

- channel reservation [1], [10], [11], [13],

- queuing of hand-off requests [2], [8],

- channel rearrangement [14].

While the strategies of enqueing hand-off requests and that of rearranging channels, especially in dynamic channel allocation environments, are worthwhile and can reduce the $\mathrm{CDP}$, in this work, we only look at QoS provisioning schemes that reserve resources in cells on behalf of mobile hosts in anticipation of their arrival. Being, at the same time, simple and natural, the resource reservation problem has recently received well-deserved attention. We refer the reader to [13] and [16] for surveys of recent literature.

There are, essentially, two approaches to resource reservation:

- fixed reservation-where a certain percentage of the available resources in a cell are permanently reserved for hand-off connections, and

- statistical reservation-where resources are reserved using a heuristic approach. These approaches range from allocating the maximum of the resource requirements of all connections in neighboring cells to reserving only a fraction of this amount [10], [13].

\subsection{Our Contributions}

The main contribution of this paper is to propose a novel, rate-based, borrowing scheme for QoS provisioning in highspeed cellular networks carrying multimedia traffic. Key to our approach are a resource reservation scheme and a companion fair borrowing scheme. At call setup time, the connections are expected to specify 1) their desired amount of bandwidth and 2) the minimum amount of bandwidth needed to ensure an adequate level of quality. Our scheme attempts to allocate the desired bandwidth to every multimedia connection originating in a cell or being handed off to that cell.

The novelty of our scheme is that, in the case of insufficient bandwidth, in order not to deny service to a requesting connection (new or hand-off), bandwidth will be borrowed on a temporary basis from existing connections. Our borrowing scheme guarantees that no connection will give up more than its fair share of bandwidth in the sense that the amount of bandwidth borrowed from a connection is proportional to its tolerance to bandwidth loss.

There are four important points to note about our scheme that we detail next:

1. First, our scheme guarantees that the bandwidth allocated to a real-time connection never drops below the minimum bandwidth requirement specified by the connection at call setup time. This is very critical to ensuring that the corresponding application can still function at an acceptable level.

2. Second, our scheme guarantees that, if bandwidth is borrowed from a connection, it is borrowed in small increments, allowing time for application-level adaptation.

3. Third, our borrowing scheme is fair in the sense that if bandwidth is borrowed from one connection, it is also borrowed from the existing connections. Specifically, if borrowing is necessary in order to accommodate a requesting connection (new or hand-off), every existing connection will give up bandwidth in proportion to its tolerance to bandwidth loss. This motivated us to refer to our scheme as rate-based fair.

4. Finally, the borrowed bandwidth is returned to the connections as soon as possible. Thus, the degradation in the QoS is transient and limited to a minimum. 
Extensive simulation results show that our rate-based QoS provisioning scheme outperforms the best previously known schemes in terms of call dropping probability and call blocking probability. In addition, our scheme ensures a high bandwidth utilization in the cellular system.

The remainder of this work is organized as follows: Section 2 reviews relevant results from the literature. Section 3 discusses the details of our rate-based QoS provisioning scheme. To begin, Section 3.1 describes the assumed cell and connection parameters. Section 3.2 further discusses the fairness of our scheme. Section 3.3 presents the details of the new call admission protocol. Section 3.4 gives the details of the hand-off management protocol. Section 3.5 shows that, in our scheme, connections recover in a timely manner after hand-off. Section 4 gives a detailed description of our simulation model. The experimental results obtained from extensive simulations are presented in Section 5. Finally, Section 6 offers concluding remarks and points out directions for further work.

\section{State of the Art}

In order to set the stage for our rate-based QoS provisioning scheme, we now briefly review the bandwidth allocation and reservation schemes proposed in [13]. We chose these schemes as a benchmark since they are arguably better than other comparable bandwidth allocation and reservation schemes found in the literature [13].

The traffic offered to the cellular system is assumed to belong to two classes:

1. Class I traffic-real-time multimedia traffic, such as interactive voice and video applications, and

2. Class II traffic-non-real-time data traffic, such as e-mail or ftp.

When a mobile host requests a new connection in a given cell, it provides the following parameters:

- the desired class of traffic (either I or II),

- the desired amount of bandwidth for the connection, and

- the minimum acceptable amount of bandwidth, that is, the smallest amount of bandwidth that the source requires in order to maintain acceptable quality, e.g., the smallest encoding rate of its codec.

One of the significant features of the call admission control and bandwidth reservation schemes in [13] is that, in order to admit the connection, bandwidth must be allocated in the originating cell and, at the same time, bandwidth must be reserved for the connection in all the neighboring cells. Specifically, for a new connection to be admitted in a cell, the cell must be able to allocate to the connection its desired bandwidth. For Class I connections, the call will be blocked unless the desired bandwidth can be allocated to it in the original cell and some bandwidth can be reserved for it in each of its six neighboring cells.

During a hand-off, an established Class I connection is dropped if its minimum bandwidth requirement cannot be met in the new cell or if appropriate reservations cannot be made on its behalf in the new set of neighboring cells. However, Class II traffic has no minimum bandwidth requirement in the case of a hand-off and a call will be continued if there is any free bandwidth available in the new cell.

Numerous approaches for reserving bandwidth have been reported in the literature [1], [2], [9], [10], [11], [13], [16]. The schemes presented in [13] use statistical reservation techniques based on the number of connections in neighboring cells, the size of the connections in neighboring cells, the predicted movement of mobile hosts, and combinations of these factors. It is worth noting that the reservation schemes in [13] keep the dropping probability for Class I connections very low since the mobile host should find bandwidth reserved for it, regardless of the cell to which it moves. But, bandwidth may be wasted in the neighboring cells (the host can only move to one neighbor) and the blocking probability in those cells may increase because unused bandwidth is being kept in reserve. In general, the schemes described in [13] favor minimizing the CDP at the expense of the CBP and give Class I traffic precedence over Class II traffic.

\section{The Rate-Based Borrowing Scheme}

It is clear that keeping a small pool of bandwidth always reserved for hand-offs, as in [13], yields low CDP. However, in our scheme, the size of the reserved pool is not determined by requests from neighboring cells, but is fixed at a certain percentage of the total amount of bandwidth available in the cell. We found that this produced results similar to the best results reported in [13] without the overhead of communication between neighboring base stations to request and release reservations. To further reduce the CDP in our scheme, we treat the reserved pool very carefully. We do not allow bandwidth from the reserved pool to be allocated to incoming hand-offs unless the bandwidth is needed to meet the minimum bandwidth requirements of the connection. Like [13], our scheme gives precedence to Class I connections; Class II traffic does not make use of the reserved bandwidth. In order to lower the call blocking probability as well as the dropping probability, our scheme allows for borrowing resources (i.e., bandwidth) from existing connections. Our borrowing strategy has the following interesting features:

1. No Class I connection will ever have to give up bandwidth beyond the minimum level negotiated at call setup time.

2. If the cell does not have enough residual bandwidth to accommodate an incoming call, the existing connections will temporarily have to give up a certain amount of bandwidth (see Section 3.3 for details).

3. If bandwidth must be borrowed, it is borrowed gradually in small increments to allow time for application-level adaptation.

4. As soon as bandwidth becomes available due to a terminating call or to a mobile host leaving the cell, the borrowed bandwidth will be returned to the degraded connections. 


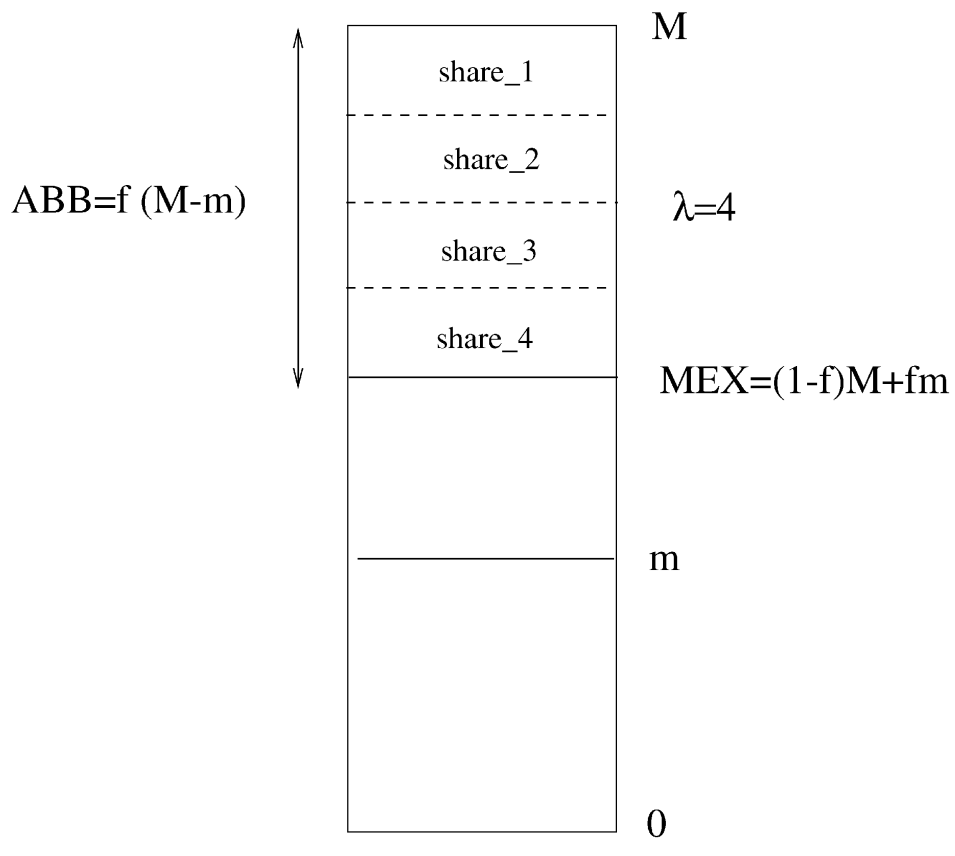

Fig. 1. Illustrating the main connection parameters.

5. Our scheme is fair in the sense that if bandwidth is borrowed, all connections will give up an amount of bandwidth proportional to their tolerance to bandwidth loss.

6. Our scheme only requires minimal computational overhead and no communication overhead.

\subsection{Cell and Connection Parameters}

Each cell maintains a pool of bandwidth reserved for Class I hand-offs which, initially, represents $r$ percent of the total bandwidth. At setup time, each connection specifies to the cell in which it originates a maximum bandwidth $M$ (termed the desired bandwidth) and a minimum bandwidth $m$, as illustrated in Fig. 1. The difference between these two values is the bandwidth_loss tolerance (BLT) of the connection. Thus,

$$
B L T=M-m .
$$

We note that, for constant bit rate (CBR) connections, $M=m$, indicating no bandwidth_loss tolerance and, thus, $B L T=0$.

Each cell maintains a local parameter, $f(0 \leq f \leq 1)$, which represents the fraction of the $B L T$ that a connection may have to give up in the worst case. This fraction is the actual borrowable bandwidth (ABB) of the connection. Thus,

$$
A B B=f \times B L T=f(M-m) .
$$

By accepting a new call, the cell agrees that the supplied bandwidth will not fall below a certain level that we call the minimum expected (MEX) bandwidth that the connection is guaranteed to receive during its stay in its starting cell. By definition, $M E X=M-A B B$. It is worth noting that $M E X \geq m$. Simple computation shows that MEX is a weighted average of $M$ and $m$ in the sense that

$$
M E X=(1-f) \cdot M+f \cdot m .
$$

To prevent borrowing from producing noticeable changes in a connection's QoS, we introduce another cell parameter, $\lambda$. The ABB is divided into $\lambda$ shares, each share being equal to $\frac{M-m}{\lambda}$. This provides the basis for a method of borrowing bandwidth gradually from a set of connections whose allocated resources may be quite different. A cell is said to be operating at level $L(0 \leq L \leq \lambda)$ when all its ongoing connections have had $L$ (or more) shares borrowed from them.

It is important to note, however, that it is possible for a connection to be missing more than $L$ shares after a handoff due to the sacrifices made to prevent call dropping. However, our scheme attempts to restore bandwidth to hand-off connections as soon as it becomes available. We refer the reader to Appendix A for the pseudocode of the initialization protocol needed to begin QoS negotiations.

\subsection{Fairness of the Borrowing Scheme}

We now introduce a further connection parameter that we call adaptivity (AD), which underlies our borrowing scheme. Specifically, for a given connection, $\mathrm{AD}$ is the ratio between the connection's bandwidth loss tolerance and the maximum bandwidth that the connection can use.

$$
A D=\frac{\text { bandwidth_loss_tolerance }}{\text { desired_bandwidth }}=\frac{M-m}{M} .
$$

It is worth noting that the higher the $\mathrm{AD}$ the more adaptive the connection and the lower the probability of a forced termination in case of a hand-off. Notice again that, for CBR connections, the adaptivity is 0 .

Consider an arbitrary cell operating at level $L$. Recall that this implies that every connection in the cell has given up $L$ of its shares. Consider an arbitrary connection $C$ with desired and minimum bandwidth $M$ and $m$, respectively. Since the cell operates at level $L$, connection $C$ must have lost $L$ of its shares operating at an effective bandwidth of

$$
M-L \times \frac{A B B}{\lambda} .
$$


The loss ratio (LR) of connection $C$ is the ratio between the amount of bandwidth borrowed from $C$ and the maximum bandwidth $M$ specified by $C$ at setup time. In other words,

$$
L R=\frac{L \times \frac{A B B}{\lambda}}{M} .
$$

Direct manipulations of (2) reveal that

$$
L R=\frac{L f}{\lambda} \times \frac{M-m}{M}=\frac{L f}{\lambda} \times A D .
$$

Since, for a given cell and a given point in time, $\frac{L f}{\lambda}$ is a constant, (3) shows that the connection will give up an amount of bandwidth proportional to its adaptivity.

Let $C^{\prime}$ be a arbitrary connection in the same cell as $C$ and let $L R(C)$ and $L R\left(C^{\prime}\right)$ be the corresponding loss ratios. Then, (3) allows us to write

$$
\frac{L R(C)}{L R\left(C^{\prime}\right)}=\frac{\frac{L f}{\lambda} \times A D(C)}{\frac{L f}{\lambda} \times A D\left(C^{\prime}\right)}=\frac{A D(C)}{A D\left(C^{\prime}\right)} .
$$

Thus, the ratio of the loss ratios of two connections is invariant to $L$ and is only a function of the adaptivity of the connections. This is the sense in which we consider our borrowing scheme to be fair.

\subsection{New Call Admission Protocol}

When a new call requests admission into the network in a cell operating at level $L$, the cell first attempts to provide the connection with an amount of bandwidth equal to its desired bandwidth minus $L$ shares of its $A B B$, that is

$$
M-L \cdot \frac{A B B}{\lambda}=\left(1-\frac{L f}{\lambda}\right) \cdot M+\frac{L f}{\lambda} \cdot m .
$$

If the amount of bandwidth specified in (4) exceeds the amount of bandwidth available, the cell tests to see if the call could be admitted if the cell progressed to level $L+1$. If transition to level $L+1$ will provide enough bandwidth to admit the call, the bandwidth is borrowed, the level is incremented, and the call is admitted; otherwise, the call is blocked. When the cell is operating at level $L=\lambda$, no more borrowing is allowed. It is important to note that our scheme never borrows from CBR connections or from connections that have already lost more than $L$ shares.

Every time bandwidth becomes available in a cell due to a connection releasing its bandwidth allocation, the cell will attempt to make a transition to the next lower level. As a result, the available bandwidth is returned to the connections that have lost bandwidth due to borrowing. All fluctuations in a connection's allocated bandwidth are gradual as only one share can be borrowed or returned at a time. We refer the reader to Appendix B for the pseudocode for new call admission.

\subsection{Hand-Off Management}

The hand-off admission policies differentiate between Class I and Class II connections. The reserved bandwidth is used only for Class I connections, which are admitted only if their minimum bandwidth needs can be met. When a Class I connection requests admission into a cell as a hand-off, the cell checks to see if the minimum bandwidth requirement can be met with the sum of the available free and reserved bandwidth in the cell. If such is the case, the call is admitted into the cell and given bandwidth from the free bandwidth up to its desired level minus $L$ shares. The connection is given bandwidth from the reserved bandwidth pool only if it is needed to reach its minimum requirement. If the minimum cannot be met using the free and reserved bandwidth, the cell tests to see if scaling to level $L+1$ would free up enough bandwidth to admit the call. If so, the cell scales the other calls in the cell and provides the hand-off call with bandwidth according to the guidelines described above.

On the other hand, Class II traffic will only be dropped if there is no free bandwidth left in the cell at all. The reserved pool is not available to these connections because, as in [13], we assume that Class II traffic is able and willing to incur a possibly substantial fluctuation in service rather than be disconnected. Calls that have suffered a lowering of bandwidth due to a hand-off will eventually be brought back to a reasonable level as their new cell has free bandwidth to give them. This is in sharp contrast to the schemes presented in [13], which have no facility to improve connections which have been degraded due to a hand-off. We refer the reader to Appendix C for the pseudocode for hand-off management.

\subsection{How Well Does a Hand-Off Do?}

Recall that a hand-off Class I connection may be cut down to its minimum in order to avoid dropping the call. In addition, our scheme specifically disallows borrowing from connections that are below the cell level $L$. When bandwidth becomes available, our scheme attempts to bring all Class I connections to the cell level $L$. In particular, this means that hand-off connections are expected to recover from a bandwidth loss incurred at hand-off time.

Fig. 2 is illustrating this recovery process by plotting the bandwidth allocated to a Class I hand-off connection over time. At time 0 , the connection is admitted into the cell at its minimum acceptable level. In roughly 35 time units (seconds in our simulation), the bandwidth has been replenished to the cell level. That the connection has reached the cell level $L$ is evident from the fact that the connection is borrowed ${ }^{2}$ from.

\section{Simulation Model}

In order to evaluate the performance of our rate-based borrowing scheme, we implemented and simulated two other schemes for comparison. First, we implemented a request-based statistical reservation scheme from [13], termed the uniform and bandwidth-based model. According to this scheme, when reservations are made on behalf of a connection in neighboring cells, an equal amount of bandwidth is reserved in each neighboring cell with no consideration of the most likely cell to which the host might travel. A cell does not reserve the sum of all the bandwidth it is asked to reserve, but just the largest of all the current requests.

2. In Fig. 2, this shows as a small decrease in bandwidth. 


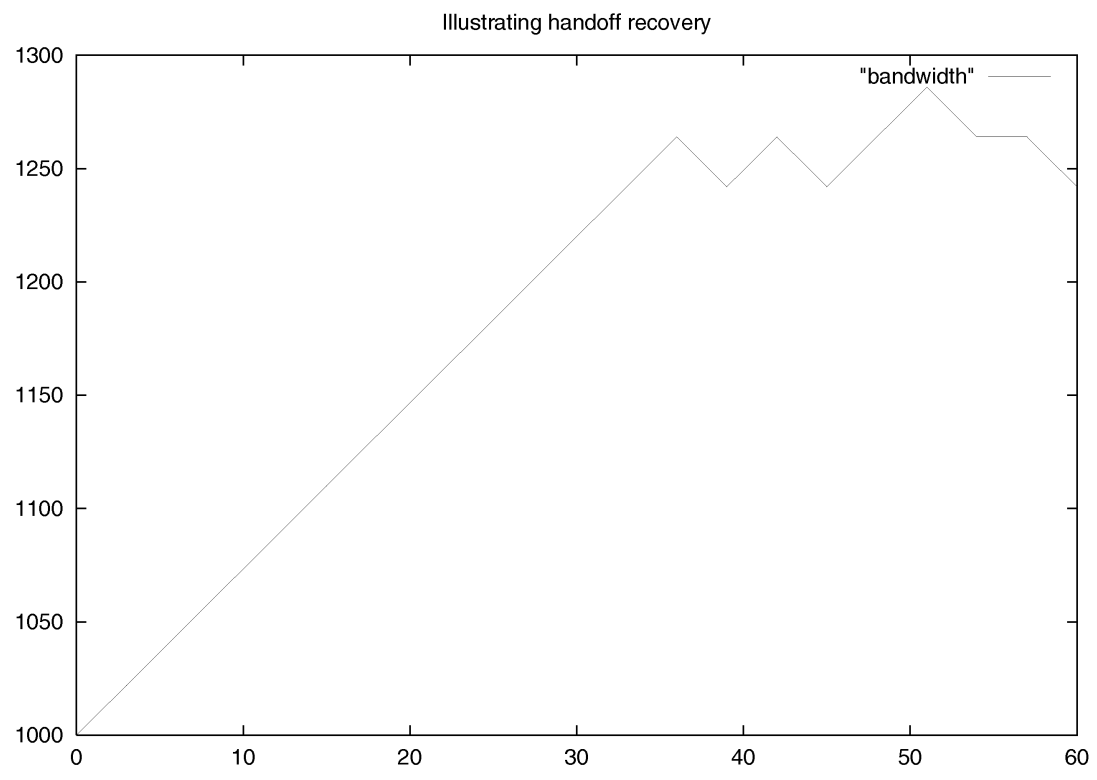

Fig. 2. Illustrating the recovery of a Class I hand-off.

We also simulated a simple scheme that reserves 5 percent of the total bandwidth in each cell for hand-offs. New calls are admitted into the network if their desired bandwidth can be met; otherwise, they are blocked. Class I hand-offs are admitted if at least their minimum bandwidth requirements can be met. They are only given enough bandwidth from the reserved pool to meet their minimum if there is too little free bandwidth available. Class II hand-offs are admitted if there is any free bandwidth in the cell.

To simulate our rate-based borrowing scheme, we used a fixed reservation pool representing 5 percent of the total bandwidth. We set $f$ to 0.5 , thus permitting borrowing up to half of the bandwidth_loss tolerance. And, we set $\lambda$ to 10 so that each call had 10 shares to give.

To fairly contrast our scheme to the one in [13], we used the traffic types and characteristics given in [13] and modeled traffic behavior just as described there with the exception of the hand-offs. In [13], a hand-off would occur during a connection with some given probability and that probability would decrease exponentially with each successive hand-off during the connection. We chose a different approach that seemed more realistic. We gave each mobile host a speed characteristic specifying the amount of time that will be spent in each cell during a call. Thus, longer calls are likely to experience more hand-offs than shorter ones. Even with this minor change, our results for the scheme from [13] correspond very closely to the results given there.

Table 1 shows the exact characteristics of the traffic used in our model. Each of the six types occurs with equal probability. For the results discussed in the following section, the speed was set to a host spending from 1 to 15 minutes in a cell with an average of 5 minutes per cell. Each cell has $30 \mathrm{Mbps}$ of bandwidth. The network is a hexagonal grid of size $6 \times 6$ consisting of 36 cells. Traffic is provided to each cell at the level being measured. If a host moves out of the $6 \times 6$ grid, it is as though the connection ended normally-hosts do not "bounce" back into the network.

\section{Experimental Results}

Fig. 3 compares the values of bandwidth utilization for the request-based reservation scheme from [13] for a fixed reservation scheme with $r=5$ percent and for our ratebased borrowing scheme with $r=5$ percent, $\lambda=10$, and $f=0.5$ so that at most half of a call's bandwidth_loss tolerance can be borrowed. For the fixed reservation scheme and the rate-based borrowing scheme at the maximum connection rate, the bandwidth utilization comes close to

TABLE 1

Traffic Characteristics for Our Simulation Model

\begin{tabular}{|l|l|l|l|l|l|l|}
\hline CLASS & AVG BPS & MIN BPS & MAX BPS & AVG CALL & MIN CALL & MAX CALL \\
\hline Class I & $30 \mathrm{Kbps}$ & $30 \mathrm{Kbps}$ & $30 \mathrm{Kbps}$ & $180 \mathrm{~s}$ & $60 \mathrm{~s}$ & $600 \mathrm{~s}$ \\
\hline Class I & $256 \mathrm{Kbps}$ & $256 \mathrm{Kbps}$ & $256 \mathrm{Kbps}$ & $300 \mathrm{~s}$ & $60 \mathrm{~s}$ & $1800 \mathrm{~s}$ \\
\hline Class I & $3000 \mathrm{Kbps}$ & $1000 \mathrm{Kbps}$ & $6000 \mathrm{Kbps}$ & $600 \mathrm{~s}$ & $300 \mathrm{~s}$ & $18000 \mathrm{~s}$ \\
\hline Class II & $10 \mathrm{Kbps}$ & $5 \mathrm{Kbps}$ & $20 \mathrm{Kbps}$ & $30 \mathrm{~s}$ & $10 \mathrm{~s}$ & $120 \mathrm{~s}$ \\
\hline Class II & $256 \mathrm{Kbps}$ & $64 \mathrm{Kbps}$ & $512 \mathrm{Kbps}$ & $180 \mathrm{~s}$ & $30 \mathrm{~s}$ & $36000 \mathrm{~s}$ \\
\hline Class II & $5000 \mathrm{Kbps}$ & $1000 \mathrm{Kbps}$ & $10000 \mathrm{Kbps}$ & $120 \mathrm{~s}$ & $30 \mathrm{~s}$ & $1200 \mathrm{~s}$ \\
\hline
\end{tabular}




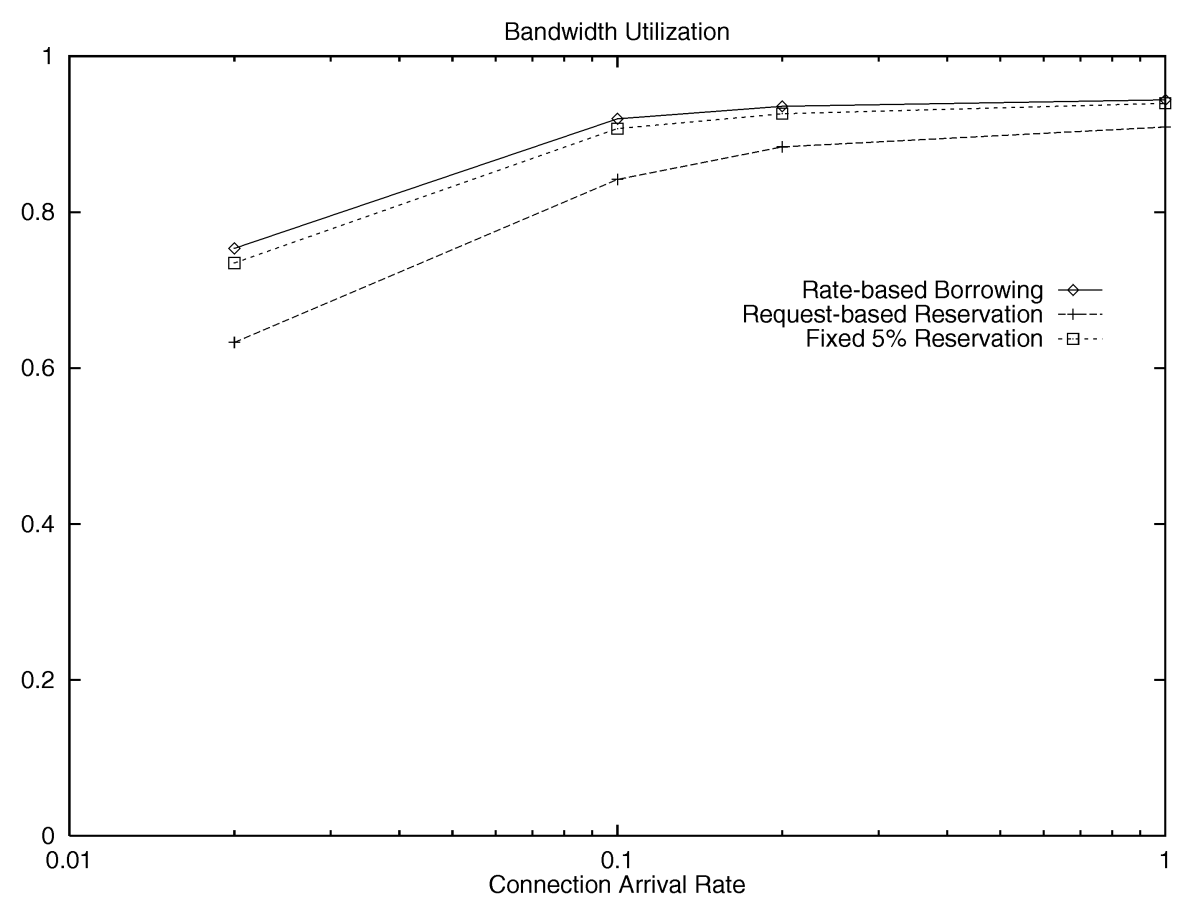

Fig. 3. A comparison of bandwidth utilization by the three schemes.

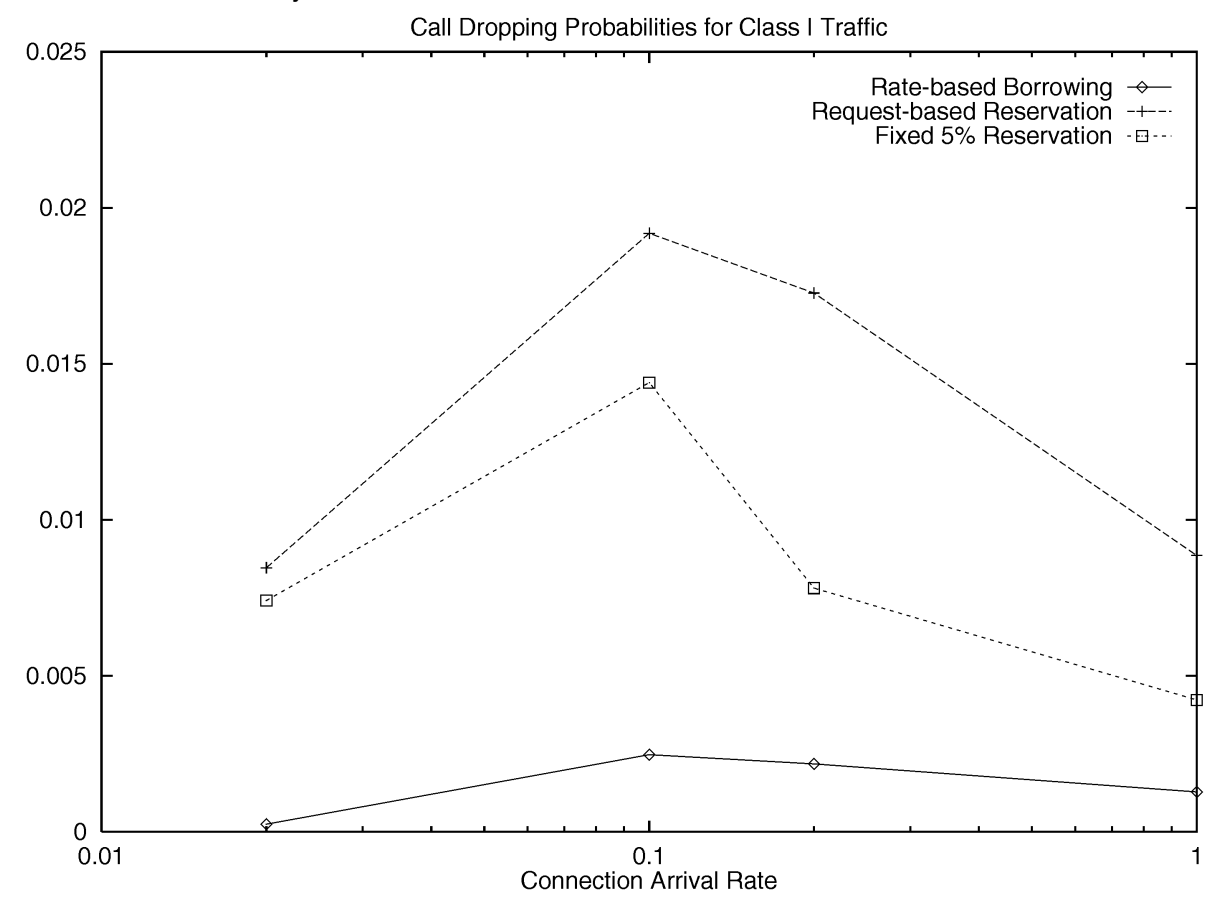

Fig. 4. Illustrating call dropping probabilities for Class I traffic.

equaling the bandwidth outside of the reserved pool. The results for the request-based reservation scheme are worse than for the other two because we did not implement a cap on the size of the reserved pool.

Figs. 4 and 5 show, respectively, the CDP for Class I traffic alone and for Class I and II traffic combined. The borrowing scheme outperforms the other two schemes in both cases. In fact, the dropping probability for Class I connections is very close to zero. The motivation, of course, for favoring Class I connections by giving them exclusive use of the hand-off reserves is that real-time connections would suffer an actual loss by being dropped. We assume that a Class II application, although inconvenienced by being dropped, would be able to resume its transmission at a later time without any significant loss. Despite this, Class II traffic fares significantly better under our ratebased borrowing scheme than under the others; it is especially important that our scheme returns bandwidth to connections who have suffered cuts during a hand-off. The values chosen for $r, f$, and $\lambda$ do have a marked impact on the results. Some of our future research will involve finding optimal values for these parameters, understanding how they relate to each other and to the QoS parameters, 


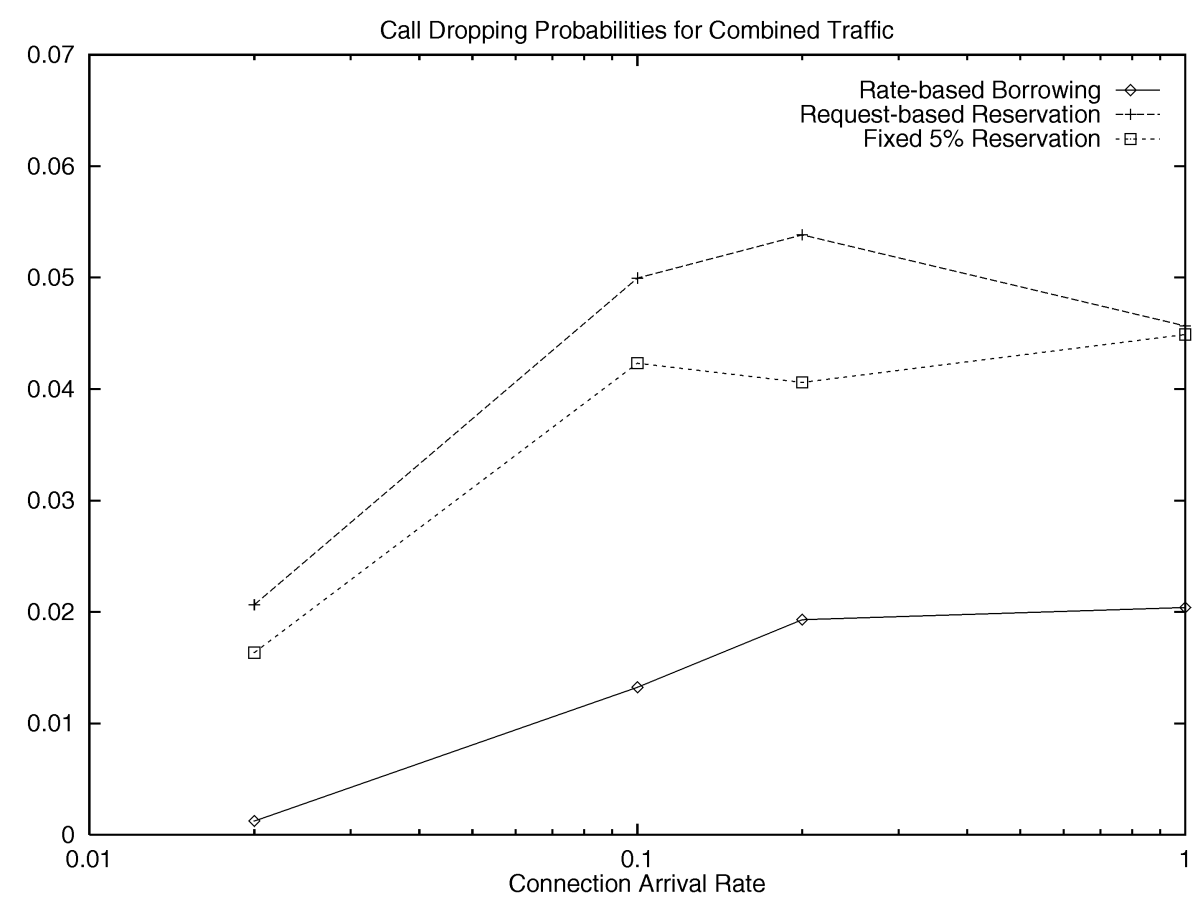

Fig. 5. Illustrating call dropping probabilities for Class I and Class II traffic combined.

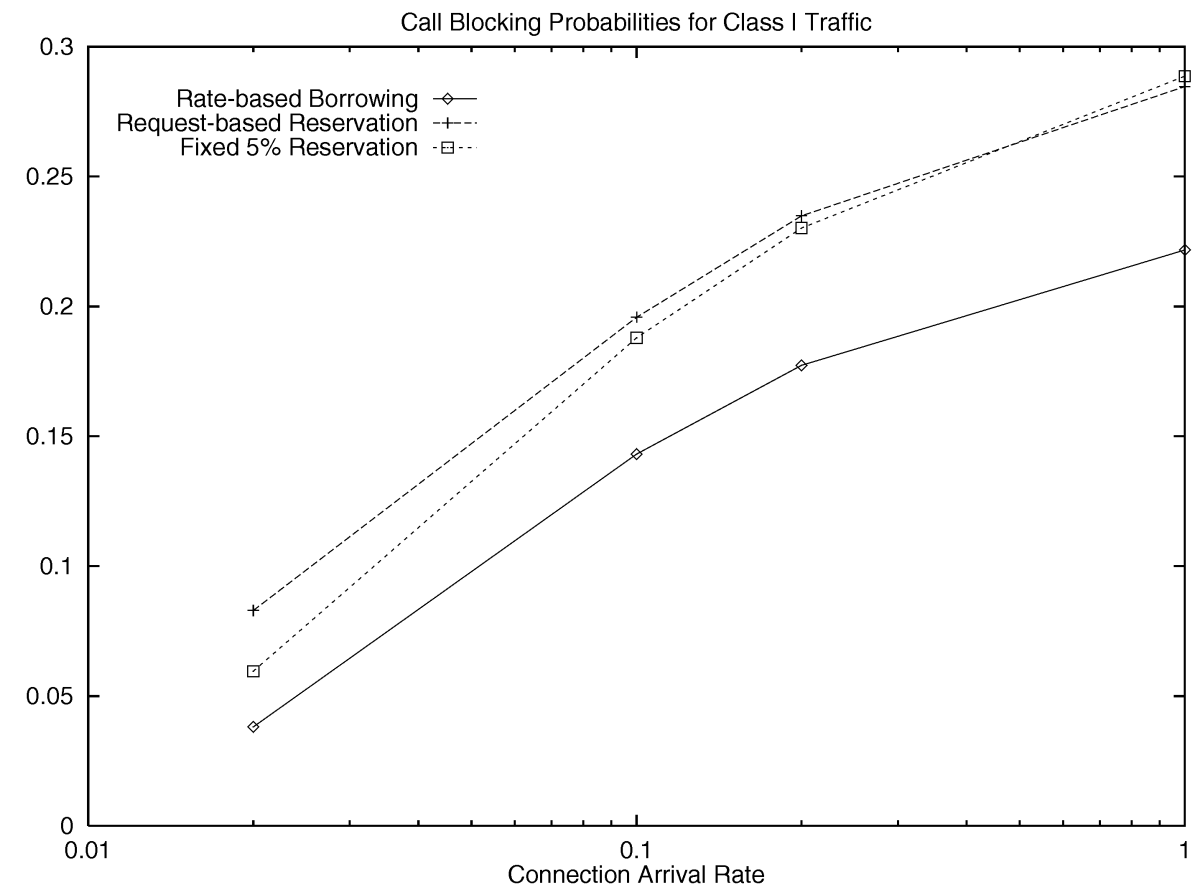

Fig. 6. Illustrating call blocking probabilities for Class I traffic.

and determining whether they can be adjusted dynamically to further increase network performance.

Next, Figs. 6 and 7 illustrate the call blocking probabilities for Class I traffic alone and for Class I and II traffic combined, respectively. They demonstrate how borrowing allows a significant improvement in the CBP while also improving the dropping probability. As with CDP, the combined traffic also fares worse than Class I traffic alone in terms of CBP. However, this is not due to any bias in the algorithms, but rather to the characteristics of the traffic being simulated. The Class II traffic requires more bandwidth on average.
But, all this success does not come without a price. Bandwidth borrowing subjects connections to possibly frequent fluctuations in the amount of bandwidth they are provided. It also decreases the probability that calls will always be provided their desired amount of bandwidth. In the two comparison schemes, the only fluctuation in bandwidth and the only possibility of a reduction in the desired amount would occur due to a hand-off. In a simulation run at a rate of one connection per second, we found that the bandwidth supplied to a connection fluctuated an average of once every 10 seconds. We saw that the calls that lost bandwidth during a hand-off 


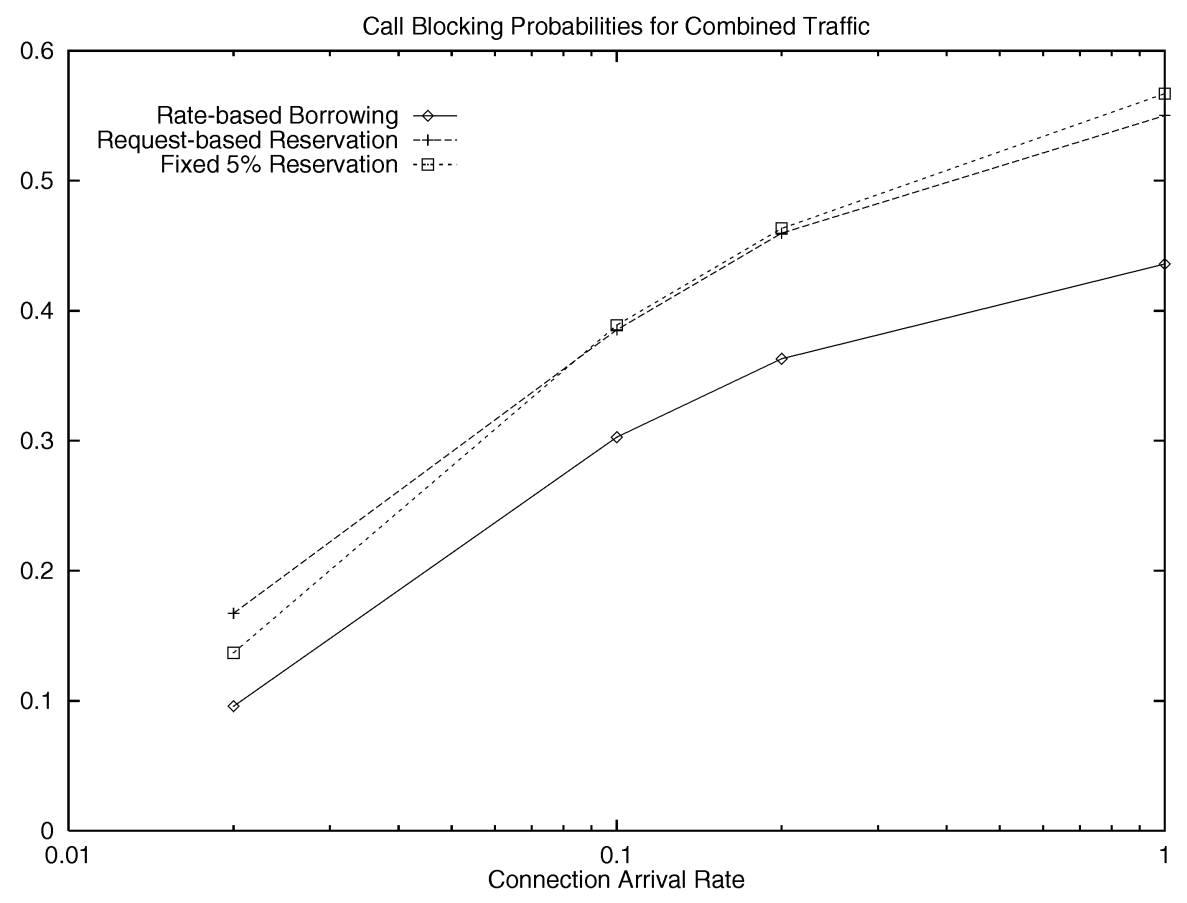

Fig. 7. Illustrating call blocking probabilities for Class I and Class II traffic combined.

were always steadily replenished. However, the networkwide average bandwidth provided to each call was about 85 percent of the desired amount. It is clear that this issue requires more research-maybe a new QoS parameter quantifying the consistency of a connection and what trade-offs between this new parameter and the other parameters are useful and tolerable.

\section{Concluding Remarks and Directions for FUTURE WORK}

Recent advances in wireless communications have presented cellular networks with the challenge of supporting real-time interactive multimedia traffic such as video tele-conInitializationferencing. However, mobility of hosts and scarcity of bandwidth makes QoS provisioning a difficult task in cellular networks.

Since multimedia traffic is intended mainly for human consumption [15] and since human senses are most forgiving, multimedia applications can tolerate and gracefully adapt to transient fluctuations in the QoS that they receive from the network. We have demonstrated that the additional flexibility afforded by the ability of multimedia applications to tolerate and adapt to transient changes in the QoS parameters can be exploited by protocol designers to significantly improve the overall performance of wireless systems.

Our main contribution is a novel, rate-based, borrowing scheme for QoS provisioning in high-speed cellular networks carrying multimedia traffic. To the largest extent possible, our scheme attempts to allocate the desired bandwidth to every multimedia connection originating in a cell or being handed off to that cell. The novelty of our scheme resides in the fact that, in the case of insufficient bandwidth, in order not to deny service to requesting connections (new or hand-off), bandwidth is borrowed on a temporary basis from existing connections.
One important characteristic of our rate-based borrowing scheme is that no connection gives up more than its "fair share" of bandwidth in the sense that the amount of bandwidth borrowed is proportional to the connection's tolerance to bandwidth loss. Importantly, our scheme ensures that the borrowed bandwidth is returned promptly to the connections.

Extensive simulation results reveal that our scheme features very low call dropping probability, low call blocking probability, good bandwidth utilization, and reasonable success with keeping both classes of connections operating steadily near their desired bandwidth.

But, our successes do not come without a price. Bandwidth borrowing subjects connections to possibly frequent fluctuations in the amount of bandwidth they are provided. In the two comparison schemes, the only fluctuation in the bandwidth provided would occur due to a hand-off. In a simulation run at a rate of one connection per second, we found that the bandwidth supplied to a connection fluctuated an average of once every 10 seconds. It is clear that this issue requires more research.

Bandwidth borrowing decreases the probability that calls will always be provided their desired amount of bandwidth. In simulation, we noticed that the calls that lost bandwidth during a hand-off were always steadily replenished; however, the network-wide average bandwidth provided to each call was about 85 percent of the desired amount. In the case of gracefully adaptive multimedia applications, it makes sense to introduce a novel QoS parameter that specifies, for each connection, the fraction of time that it can tolerate operating below its expected bandwidth level. We are working on incorporating such a QoS parameter into cellular networks. This promises to be an exciting area for further research. 


\section{APPENDix A}

\section{INITIALIZATION-PSEUDOCODE}

Protocol Initialize

call_ABB =

(call_desired_bw-call_min_bw) * $f$;

// Find the size of a share for this call

call_share $=$ call_ABB $/ \lambda$;

// Scale down the desired bandwidth to the

operating level $L$ of the cell

call_scaled_bw = call_desired_bw -

$L$ * call\_share;

\section{APPENDIX B}

\section{New Call Admission-Pseudocode}

\section{Protocol Admit_new-connection}

//Total the values of one share of each existing connection in the cell

one_level_bw = sum_existing_calls ( ); free_bw = total_bw - bw_used - bw_reserved;

if ( call_scaled_bw $\leq$ free_bw) call is ACCEPTED;

else if ( $L<\lambda$ AND call_scaled_bw call_share $\leq$ free_bw + one_level_bw)

\{

//if the cell is not yet at its last level

//AND the call will fit after a round of borrowing

call is ACCEPTED;

// scale down

scale_down_existing_connections () ;

$\mathrm{L}=\mathrm{L}+1$;

call_scaled_bw = call_scaled_bw call_share;

\}

else

call is BLOCKED

if ( call is accepted)

call_granted_bw =call_scaled_bw;

\section{APPENDIX C}

\section{HANDoff MANAGement-Pseudocode}

\section{Protocol Admit_hand-off_connection}

one_level_bw = sum_existing_calls ( ) ;

free_bw = total_bw - bw_used - bw_reserved; if ( call is CLASS I )

\{

if ( call_min_bw $\leq$ free_bw + reserved_bw)

// Reserved bandwidth is available to

this connection

call is ACCEPTED;

else if ( $L<\lambda$ AND call_min_bw $\leq$ free_bw +

reserved_bw + one_level_bw)

\{

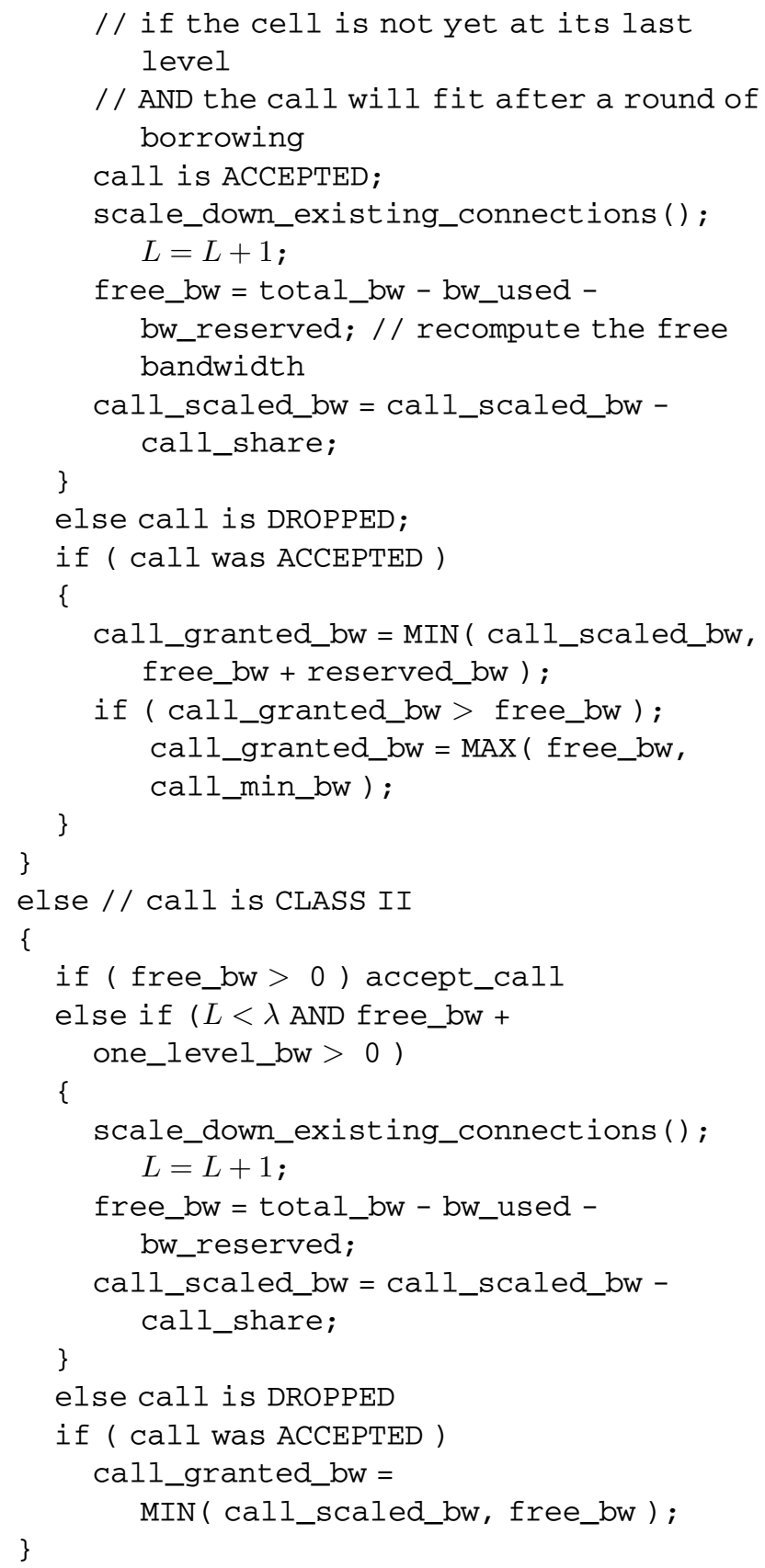

// if the cell is not yet at its last level

/ / AND the call will fit after a round of borrowing

call is ACCEPTED;

scale_down_existing_connections () ; $L=L+1$;

free_bw = total_bw - bw_used -

bw_reserved; / / recompute the free bandwidth

call_scaled_bw = call_scaled_bw cal1_share;

\}

else call is DROPPED;

if ( call was ACCEPTED)

\{

call_granted_bw $=$ MIN ( call_scaled_bw free_bw + reserved_bw ) ;

if ( call_granted_bw $>$ free_bw ) ; call_granted_bw $=$ MAX ( free_bw, call_min_bw );

\}

\}

else / / call is CLASS II

\{

if ( free_bw $>0$ ) accept_call

else if ( $L<\lambda$ AND free_bw +

one_level_bw $>0$ )

\{

scale_down_existing_connections () ; $L=L+1$;

free_bw = total_bw - bw_used -

bw_reserved;

call_scaled_bw = call_scaled_bw cal1_share;

\}

else call is DROPPED

if ( call was ACCEPTED)

call_granted_bw =

MIN ( call_scaled_bw, free_bw ) ;

\}

\section{ACKNOWLEDGMENTS}

The authors wish to express their thanks to the four anonymous referees for their very detailed comments that have contributed to a better presentation of the results in this work. This work was supported by US Office of Naval Research grant N00014-97-1-0562.

\section{REFERENCES}

[1] A. Acampora and M. Naghshineh, "Control and Quality-ofService Provisioning in High-Speed Microcellular Networks," IEEE Personal Comm., vol. 1, second quarter 1994.

[2] P. Agrawal, D.K. Anvekar, and B. Narendran, "Channel Management Policies for Handovers in Cellular Networks," Bell Labs Technical J., vol. 1, pp. 96-109, 1996. 
[3] R. Bolla, F. Davoli, and M. Marchese, "Bandwidth Allocation and Admission Control in ATM Networks with Service Separation," IEEE Comm., pp. 130-137, 1997.

[4] C.-C. Chao and W. Chen, "Connection Admission Control for Mobile Multiple-Class Personal Communication Networks," IEEE J. Selected Areas in Comm., vol. 15, pp. 1618-1626, 1997.

[5] S. Chen and K. Nahrstedt, "Distributed Quality-of-Service Routing in Ad-Hoc Networks," IEEE J. Selected Areas in Comm., vol. 17 pp. 1488-1505, 1999.

[6] M.-H. Chiu and M.A. Bassiouni, "Predictive Schemes for Handoff Prioritization in Cellular Networks Based on Mobile Positioning," IEEE J. Selected Areas in Comm., vol. 158, pp. 510-522, 2000.

[7] B.M. Epstein and M. Schwartz, "Predictive QoS-Based Admission Control for Multi-Class Traffic in Cellular Wireless Networks," IEEE J. Selected Areas in Comm., vol. 18, pp. 523-534, 2000.

[8] D. Hong and S. Rappaport, "Traffic Model and Performance Analysis for Cellular Mobile Radio Telephone Systems with Prioritized and Non-Prioritized Handoff Procedures," IEEE Trans. Vehicular Technology, vol. 35, pp. 77-92, 1986.

[9] H. Kanakis, P.P. Mishra, and A. Reibman, "An Adaptive Congestion Control Scheme for Real-Time Video Packet Transport," IEEE/ACM Trans. Networking, vol. 3, 1996.

[10] D. Levine, I. Akyildiz, and M. Naghshineh, "A Resource Estimation and Call Admission Algorithm for Wireless Multimedia Networks Using the Shadow Cluster Concept," IEEE/ACM Trans. Networking, vol. 5, pp. 1-12, 1997.

[11] M. Naghshineh and M. Schwartz, "Distributed Call Admission Control in Mobile/Wireless Networks," IEEE J. Selected Areas in Comm., vol. 14, pp. 711-717, 1996.

[12] M. Naghshineh and M. Willebbek-LeMair, "End-to-End QoS Provisioning in Multimedia Wireless/Mobile Networks Using an Adaptive Framework," IEEE Comm., pp. 72-81, 1977.

[13] C. Oliviera, J. Kim, and T. Suda, "An Adaptive Bandwidth Reservation Scheme for High Speed Multimedia Wireless Networks," IEEE J. Selected Areas in Comm., vol. 16, pp. 858-874, 1998.

[14] S. Papavassiliou, L. Tassiulas, and P. Tandon, "Meeting QoS Requirements in a Cellular Network with Reuse Partitioning," IEEE J. Selected Areas in Comm., vol. 12, pp. 1389-1400, 1994.

[15] S.V. Raghavan and S.K. Tripathy, Networked Multimedia Systems. Prentice Hall, 1998.

[16] H.G. Perros and K.M. Elsayyed, "Call Admission Control Schemes: A Review," IEEE Comm. Magazine, pp. 82-91, 1996.

[17] L. Trajkovic and A. Neidhardt, "Effect of Traffic Knowledge on the Efficiency of Admission-Control Policies," ACM Computer Comm. Rev., pp. 5-34, 1999

[18] N. Tran and K. Nahrstedt, "Adaptive Adaptation by Program Delegation in VOD," Proc. Int'l Conf. Multimedia Computing and Systems, pp. 96-107, 1998.

[19] B.J. Vickers, M. Lee, and T. Suda, "Feedback Control Mechanism for Real-Time Multipoint Video Services," IEEE J. Selected Areas in Comm., vol. 15, 1997.

[20] W.-B. Yang and E. Geraniotis, "Admission Policies for Integrated Voice and Data Traffic in CDMA Packet Radio Networks," IEEE J. Selected Areas in Comm., vol. 12, pp. 654-664, 1994.

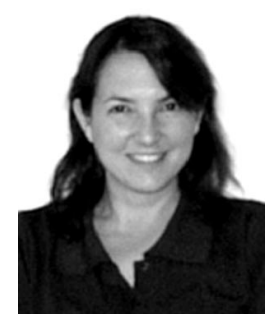

Mona El-Kadi received the BS degree in computer science from Old Dominion University (ODU) in 1985. She spent 14 years working in industry, primarily in the US Department of Defense Research and Development and in Department of Defense operations, as well as on commercial systems. Currently, she is a PhD student at ODU, doing research on QoS in cellular and satellite networks.

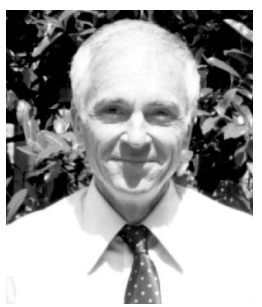

Stephan Olariu received the MSc and $\mathrm{PhD}$ degrees in computer science from McGill University, Montreal, in 1983 and 1986, respectively. In 1986, he joined the Computer Science Department at Old Dominion University, where he is now a professor. He has published extensively in various journals, book chapters, and conference proceedings. His research interests include wireless networks and mobile computing, parallel and distributed systems, performance evaluation, and medical image processing. He serves on the editorial board of several archival journals, including IEEE Transactions on Parallel and Distributed Systems, Journal of Parallel and Distributed Computing, International Journal of Foundations of Computer Science, Journal of Supercomputing, International Journal of Computer Mathematics, VLSI Design, and Parallel Algorithms and Applications. He is a member of the IEEE.

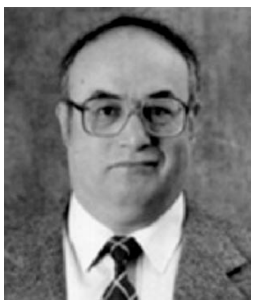

Hussein Abdel-Wahab received the BS degree in electrical engineering from Cairo University in 1969 and the MS and PhD degrees in computer communications from the University of Waterloo in 1973 and 1976, respectively. Currently, he is a full professor of computer science at Old Dominion University. In addition, he is an adjunct professor of computer science at the University of North Carolina at Chapel Hill and a research faculty member at the Information Technology Lab of the National Institute of Standards and Technology. Prior to that, he held faculty positions at North Carolina State University, the University of Maryland, and the Rochester Institute of Technology. $\mathrm{He}$ served as a consultant to many organizations, including IBM, MCNC, and MITRE Corp. He is the principle investigator in the design and implementation of XTV, a pioneer multimedia conferencing system. His main research interests are multimedia collaborative systems, internet security, and mobile communications. His research has been supported by the US National Science Foundation, the US Office of Naval Research, IBM, MCNC, MITRE, and the the US Defense Advanced Research Projects Agency among others. He is a senior member of the IEEE.

$\triangleright$ For more information on this or any computing topic, please visit our Digital Library at http://computer.org/publications/dlib. 